\title{
La flore microbienne du smen marocain I. Flore naturelle et flore d'intérêt hygiénique
}

\author{
A. EL MARRAKCHI *, A. TANTAOUI-ELARRAKI **, \\ A. EL MANE * et L. TIFRIT ${ }^{*}$ \\ * Institut Agronomique et Vétérinaire Hassan II, \\ Département d'Hygiène et Industrie des Denrées Alimentaires d'Origine Animale, \\ Rabat, Maroc \\ *** Centre National de Coordination et de Planification \\ de la Recherche Scientifique et Technique, Rabat, Maroc
}

\section{Résumé}

L'étude de la flore d'intérêt hygiénique du smen de commerce révèle que ce produit est exempt aussi bien de germes de contamination fécale que de microorganismes pathogènes tels que les staphylocoques présumés entérotoxinogènes et les salmonelles. Cette absence s'explique par les conditions dysgénésiques du milieu : salage et forte lipolyse, et montre que le smen, malgré les nombreuses manipulations que nécessite sa préparation, offre une garantie sanitaire pour le consommateur.

Au cours de l'élaboration du produit, les coliformes ne sont plus isolés dès le $40^{\mathrm{e}}$ jour alors que le nombre des entérocoques diminue. Ces observations confirment les résultats obtenus sur le produit fini.

Les micro-organismes qui forment la flore normale du smen (flore aérobie mésophile totale et flore halophile modérée), appartiennent, presque tous, au genre Bacillus et aux espèces $B$. alvei, $B$. brevis et $B$. firmus. La prédominance des Bacillus s'explique par leur résistance aux effets inhibiteurs du sel et des acides gras libres.

Mots clés : Smen - Flore naturelle - Flore d'intérêt hygiénique - Evolution au cours de la conservation.

\section{Summary}

Microbial flora of Moroccan Smen

1. Natural flora and flora of hygienic importance

The study of marketed smen for the flora of hygienic importance showed the absence of microbes of fecal contamination and pathogens such as Staphylococci and Salmonella in this product.

This is probably due to the unfavorable conditions for bacterial growth (salting and strong lipolysis) of the medium which make the smen a wholesome product despite the numerous handling steps required for its preparation. 
During smen processing, coliforms were not isolated any more by the $40^{\text {th }}$ day of processing while the number of Enterococci decreased. This supports the data previously obtained for the finished product.

The majority of microorganisms forming the normal flora of smen (Total aerobic flora and salt-tolerant flora) belongs to the Bacillus genus and particularly to B. alvei, $B$. brevis and $B$. firmus species. This is may be due to the ability of these organisms to resist to the inhibitory effects of salt and free fatty acids.

Key words: Smen - Natural flora - Flora of hygienic importance - Evolution during storage.

\section{Introduction}

L'étude chimique du smen marocain (EL MaRRaKCHI et al., 1986) montre que ce produit, au cours de son élaboration, est le siège de deux transformations importantes, l'une relative à l'hydrolyse de la matière grasse, l'autre à la baisse des teneurs en composants de la phase aqueuse (lactose et protéines).

Ces modifications chimiques, en particulier la lipolyse, sont le résultat d'activités enzymatiques d'origines microbiennes. La lipase naturelle n'a aucun effet, en raison de sa sensibilité au salage (Deeth et Firz-Gerald, 1976). Il convient donc d'analyser le rôle joué par les micro-organismes au cours des différentes transformations, en particulier, celui des micro-organismes lipolytiques et des bactéries lactiques. De plus, la préparation artisanale du smen nécessite de nombreuses manipulations qui peuvent occasionner la contamination par des bactéries dangereuses venant, ainsi, s'ajouter à celles qui ont envahi la matière première pendant les phases antérieures (traite, caillage lactique, extraction du beurre, etc.). Il est donc nécessaire d'étudier également la flore d'intérêt hygiénique.

\section{Matériel et méthodes}

Les méthodes utilisées ont pour objet l'étude quantitative et qualitative de la flore du smen. Elles nécessitent, au préalable, une préparation de l'échantillon.

\section{A. Préparation de l'échantillon}

Elle consiste en l'obtention de la matière non grasse du smen et en la réalisation des différentes dilutions.

La séparation de la phase aqueuse de la phase grasse est réalisée selon les techniques décrites par SERRES et al. (1973) pour la préparation du beurre.

La phase aqueuse sert à la préparation des dilutions.

Les dilutions successives au dixième de $10^{-1}$ à $10^{-7}$ sont réalisées dans le liquide de Ringer dilué au quart. 
Les résultats seront exprimés par $\mathrm{ml}$ de la phase non grasse sauf pour les salmonelles dont la recherche a été effectuée dans $2 \mathrm{ml}$.

\section{B. Etude quantitative}

Les dénombrements ont été réalisés dans les conditions suivantes:

- Flore mésophile aérobie totale (FMAT) et Flore psychrotrophe (FP)

- Utilisation du milieu PCA (OXOID CM 125).

- Incubation à $30^{\circ} \mathrm{C}$ pendant 3 jours pour la FMAT et $4{ }^{\circ} \mathrm{C}$ pendant 12 jours pour la FP.

- Flore halotolérante modérée (FHM)

- Utilisation du milieu PCA à $35 \%$ de chlorure de sodium.

- Incubation à $25^{\circ} \mathrm{C}$ pendant 5 jours.

- Levures et spores de moisissures.

- Utilisation du milieu PDA (OXOID CM 139).

- Incubation à $25^{\circ} \mathrm{C}$ pendant 5 jours.

- Bactéries coliformes

- Utilisation du milieu au désoxycholate lactose (MERCK 2894).

- Incubation à $30^{\circ} \mathrm{C}$ pour l'ensemble des bactéries coliformes et à $44^{\circ} \mathrm{C}$ pour les fécaux, pendant 18 à $24 \mathrm{~h}$.

- Entérobactéries

- Utilisation du milieu Mac Conkey (OXOID CM 115).

- Incubation à $30^{\circ} \mathrm{C}$ pendant 3 jours.

\section{- Entérocoques}

- Utilisation du milieu de Rothe (Institut Pasteur S 6490) et du milieu de Litsky (IPS 6486) pour confirmation.

- Incubation à $37^{\circ} \mathrm{C}$ pendant 24 à $48 \mathrm{~h}$.

- Floore anaérobie sulfito-réductrice

Utilisation de la gélose viande-foie (IPS 6457) additionnée d'alun de fer et de sulfite de sodium.

\section{- Staphylocoques pathogènes}

Leur dénombrement a nécessité trois étapes : un enrichissement, un isolement et une confirmation.

L'enrichissement est réalisé sur bouillon nutritif (OXOID CM1) à la température de $37^{\circ} \mathrm{C}$ pendant $24 \mathrm{~h}$ et l'isolement sur milieux de Chapman (OXOID CM 85) et de Baird-Parker (IPS 6481) dans les mêmes conditions d'incubation que pour l'enrichissement.

Les colonies prélevées sur les milieux d'isolement et présentant les caractères culturaux des staphylocoques présumés entérotoxinogènes font l'objet d'une confirmation de leur pouvoir pathogène par la recherche de coagulase, de la désoxyribonucléase et de la phosphatase. Ces 3 enzymes sont recherchées selon les techniques décrites par Serres et al. (1973). 


\section{- Salmonelles}

Le dénombrement des salmonelles est effectué selon les étapes suivantes : un pré-enrichissement, un enrichissement et un isolement.

Le pré-enrichissement est réalisé en ensemençant $2 \mathrm{ml}$ de la phase non grasse dans $10 \mathrm{ml}$ du bouillon au mannitol, dont la composition est la suivante : extrait de viande $1 \mathrm{~g}$, peptone $10 \mathrm{~g}$, mannitol $5 \mathrm{~g}$, chlorure de sodium $5 \mathrm{~g}$, solution alcoolique de pourpre de bromocrésol à $1,6 \%: 2 \mathrm{ml}$, eau distillée qsp $1000 \mathrm{ml}$. Le $\mathrm{pH}$ est ramené à 7 par addition de soude normale stérile.

L'incubation est effectuée à $37^{\circ} \mathrm{C}$ pendant $24 \mathrm{~h}$.

L'enrichissement est réalisé dans deux milieux différents : le bouillon au sélénite de sodium (OXOID CM 395) et le milieu au tétrathionate (OXOID CM 29), incubés respectivement à $37^{\circ} \mathrm{C}$ et $44^{\circ} \mathrm{C}$ pendant $24 \mathrm{~h}$.

Pour l'isolement, trois milieux sont simultanément utilisés: milieu DCL (OXOID CM 393), SS (OXOID CM 99) et Hektoen (OXOID CM 419). L'incubation se fait à $37^{\circ} \mathrm{C}$ pendant $24 \mathrm{~h}$. Les colonies suspectes d'être des salmonelles sont repiquées, puis font l'objet d'une confirmation selon les techniques de LE MiNOR (1972).

\section{Etude qualitative}

L'identification a concerné les souches isolées parmi les bactéries coliformes, les entérocoques, la FMAT et la FHM.

\section{Identification des coliformes}

Avant de procéder à leur identification, les colonies de coliformes ont fait l'objet d'un isolement sur le milieu EMB (OXOID CM 69).

Les colonies bien isolées et d'aspects différents sur EMB, après 18 à $24 \mathrm{~h}$ d'incubation à $37^{\circ} \mathrm{C}$, sont repiquées sur gélose nutritive inclinée en vue de leur conservation et de leur identification ultérieure.

Pour l'identification des bactéries coliformes, les techniques ont été essentiellement empruntées à Le Minor (1972) et Buttiaux et al. (1974).

Les différents milieux d'identification sont ensemencés à partir d'une culture de 18 à $24 \mathrm{~h}$ et incubés, généralement, à $37^{\circ} \mathrm{C}$.

L'identification des coliformes a été, dans certains cas, complétée par la méthode API 20E. L'utilisation des plaquettes API a été effectuée selon les indications du fabricant.

\section{Identification des entérocoques}

Les cultures positives en milieu confirmatif de Litsky sont ensemencées sur le milieu de différenciation de Barnes (IP 5323). L'incubation est réalisée à $37^{\circ} \mathrm{C}$ pendant 24 à $48 \mathrm{~h}$. 
Des colonies développées sur le milieu de Barnes sont prélevées et soumises à la coloration de Gram et à l'épreuve de la catalase pour confirmation de leur appartenance au genre Streptococcus, puis ensemencées sur bouillon lactosé et incubées à $37^{\circ} \mathrm{C}$ pendant $24 \mathrm{~h}$. Un nouveau passage sur milieu de Barnes a permis de s'assurer de la pureté de la souche.

Pour l'étude des différentes réactions, l'incubation est généralement faite à $37^{\circ} \mathrm{C}$ pendant 24 à $48 \mathrm{~h}$ sauf pour l'étude de la croissance à différentes températures. Les tests suivants ont été réalisés:

- Culture dans le lait de Sherman (lait à $0,1 \%$ de bleu de méthylène).

- Réduction du tellurite de potassium à 1 sur 2500 .

- Liquéfaction de la gélatine.

- Croissance en bouillon nutritif à $40 \%$ de bile.

- Résistance au chlorure de sodium à $6,5 \%$.

- Action sur le lait tournesolé.

- Croissance à différentes températures : 10,45 et $50^{\circ} \mathrm{C}$,

- Fermentation du lactose, saccharose, raffinose, mannitol, sorbitol et glycérol.

- Hémolyse.

\section{Identification des souches isolées parmi la FMAT et la FHM}

L'identification des bacillus a été menée selon les caractères physiologiques et biochimiques résultant des travaux de KNIGHT et Proom (1950), GORdON et al. (1973) et NoRRIs et al. (1980), celle des Micrococcaceae, qui sont minoritaires (5 souches sur 130), est réalisée jusqu'au stade du genre.

\section{Résultats}

Cette étude a concerné l'analyse du smen de commerce et du smen au cours de son élaboration.

\section{A. Etude du smen de commerce}

22 échantillons de smen, désignés de $B_{1}$ à $B_{22}$ et provenant de régions différentes (Meknès, Khouribga, Midelt, Khmisset, Salé et Rabat) ont été étudiés. A titre comparatif, deux autres échantillons, l'un de beurre fermier d'âge inconnu $\left(B_{24}\right)$ et l'autre de beurre salé importé $\left(B_{23}\right)$ ont été également analysés.

Tous les échantillons ont fait, en plus, l'objet d'une détermination de l'indice d'acide et de l'indice de peroxyde selon les techniques décrites par WOLF (1968).

\section{Aspects quantitatifs}

Les résultats des dénombrements sont consignés dans le tableau 1 . La flore de contamination globale, qui est représentée par la flore mésophile aérobie totale (FMAT), la flore psychrotrophe (FP) et la flore halotolérante 
TABLEAU 1

Dénombrement de la flore du smen* (par $\mathrm{ml}$ de la phase aqueuse)

Enumeration of the microbial flora of the smen (CFU/ml of aqueous phase)

\begin{tabular}{|c|c|c|c|c|c|c|c|}
\hline Echantillon & $\begin{array}{c}\text { Flore } \\
\text { mésophile } \\
\text { aérobie totale }\end{array}$ & $\begin{array}{c}\text { Flore } \\
\text { psychrotrophe }\end{array}$ & $\begin{array}{c}\text { Flore } \\
\text { halotolérante } \\
\text { modérée }\end{array}$ & Moisissures & Levures & Indice d'acide & $\begin{array}{l}\text { Indice de } \\
\text { peroxyde }\end{array}$ \\
\hline $\mathrm{B}_{1}$ & $4,7 \cdot 10^{2}$ & $1,8 \cdot 10^{2}$ & $1,8 \cdot 10^{2}$ & $5 \cdot 10^{4}$ & — * * * & 33,5 & 1,6 \\
\hline $\mathrm{B}_{2}$ & $2,1 \cdot 10^{=}$ & $1 \cdot 10^{2}$ & $2,3 \cdot 10^{2}$ & - & - & 34,5 & 2,2 \\
\hline $\mathrm{B}_{3}$ & $5,2 \cdot 10^{3}$ & $3,9 \cdot 10^{2}$ & $2 \cdot 10^{2}$ & $3 \cdot 10^{4}$ & $1 \cdot 10^{5}$ & 46 & 1,7 \\
\hline $\mathrm{B}_{4}$ & $2,7 \cdot 10^{2}$ & $7 \cdot 10^{1}$ & $1,8 \cdot 10^{2}$ & - & - & 44 & 1,8 \\
\hline $\mathrm{B}_{5}$ & $2,3 \cdot 10^{2}$ & $1,6 \cdot 10^{2}$ & $1,5 \cdot 10^{2}$ & - & - & 48,2 & 2,6 \\
\hline $\mathrm{B}_{6}$ & $3,6 \cdot 10^{4}$ & $4,3 \cdot 10^{2}$ & $1,2 \cdot 10^{2}$ & $1 \cdot 10^{4}$ & - & 41,2 & 1,9 \\
\hline $\mathrm{B}_{7}$ & $6 \cdot 10^{2}$ & $1,2 \cdot 10^{2}$ & $2,2 \cdot 10^{2}$ & - & - & 38,2 & 6 \\
\hline $\mathrm{B}_{8}$ & $3,2 \cdot 10^{2}$ & $5,6 \cdot 10^{2}$ & $7 \cdot 10^{2}$ & $2 \cdot 10^{4}$ & $3 \cdot 10^{5}$ & 10,4 & 1,8 \\
\hline $\mathrm{B}_{9}$ & $6,3 \cdot 10^{2}$ & $3,4 \cdot 10^{2}$ & $2 \cdot 10^{2}$ & . $10^{4}$ & - & 35,2 & 0,8 \\
\hline $\mathrm{B}_{10}$ & $1,04 \cdot 10^{4}$ & $3,7 \cdot 10^{2}$ & $1,02 \cdot 10^{2}$ & $1,9 \cdot 10^{6}$ & $1,4 \cdot 10^{6}$ & 29,9 & 4,2 \\
\hline $\mathrm{B}_{11}$ & $6,5 \cdot 10^{2}$ & $2,1 \cdot 10^{2}$ & $2,4 \cdot 10^{2}$ & $1,6 \cdot 10^{6}$ & $2 \cdot 10^{6}$ & 30,3 & 1,5 \\
\hline $\mathrm{B}_{12}$ & $8 \cdot 10^{2}$ & $1,5 \cdot 10^{2}$ & $2,8 \cdot 10^{2}$ & $7 \cdot 10^{4}$ & - $10^{5}$ & 33,6 & 2,3 \\
\hline $\mathrm{B}_{13}$ & $6,7 \cdot 10^{2}$ & $2,9 \cdot 10^{2}$ & $3,13 \cdot 10^{2}$ & $1 \cdot 10^{4}$ & . $10^{5}$ & 30 & 1,7 \\
\hline $\mathrm{B}_{14}$ & $3,03 \cdot 10^{4}$ & $6,9 \cdot 10^{2}$ & $1,2 \cdot 10^{2}$ & $7,3 \cdot 10^{6}$ & $3,4 \cdot 10^{7}$ & 52,7 & 1,7 \\
\hline$B_{15}^{14}$ & $2,71 \cdot 10^{4}$ & $1,7 \cdot 10^{2}$ & $1,86 \cdot 10^{4}$ & $7,2 \cdot 10^{6}$ & $3,7 \cdot 10^{7}$ & 51,3 & 1,52 \\
\hline $\mathrm{B}_{16}$ & $2,68 \cdot 10^{5}$ & $5,3 \cdot 10^{2}$ & $6,3 \cdot 10^{3}$ & $2 \cdot 10^{5}$ & $2,10 \cdot 10^{5}$ & 45,1 & 1,6 \\
\hline $\mathrm{B}_{17}$ & - & - & - & - & - & 52,1 & 2,4 \\
\hline $\mathrm{B}_{18}$ & - & - & - & - & - & 68,4 & 1,5 \\
\hline$B_{19}$ & - & - & - & - & - & 84,1 & 1,7 \\
\hline $\mathrm{B}_{20}$ & - & - & - & 一 & - & 82,4 & 2 \\
\hline $\mathrm{B}_{21}$ & $1,09 \cdot 10^{4}$ & $7 \cdot 10^{2}$ & $9,2 \cdot 10^{2}$ & - & - & 14,5 & 3,6 \\
\hline $\mathrm{B}_{22}$ & $1 \cdot 10^{5}$ & $4,5 \cdot 10^{3}$ & $1,4 \cdot 10^{3}$ & - & $1 \cdot 10^{4}$ & 15,7 & 3,7 \\
\hline $\mathrm{B}_{23}$ & $2,11 \cdot 10^{4}$ & $6,3 \cdot 10^{2}$ & $7,9 \cdot 10^{3}$ & - & - & 10,4 & 1,2 \\
\hline $\mathrm{B}_{24}^{(2)}$ & $2,26 \cdot 10^{6}$ & $3,9 \cdot 10^{2}$ & $1,1 \cdot 10^{3}$ & $1 \cdot 10^{4}$ & - & 5 & 4 \\
\hline
\end{tabular}

* Aucun des micro-organismes suivants n’a été mis en évidence: Coliformes totaux et fécaux, Entérobactéries, Entérocoques, Anaćrobies sulfito-réducteurs. Staphylocoques présumés pathogènes, Salmonelles et Leuconostoc. Total and fecal coliforms, Enterobacteria, Enterococci, Sulphite reducing anuerobes, presumed pathogenic Staphylococci. Salmonella and Leuconostoc species were not found in one $\mathrm{ml}$ of aqueous phase (two $\mathrm{ml}$ for salmonella).

(1) Echantillon de smen d'importation. Imported butter sample.

(2) Echantillon de beurre fermier. Local farm butter sample.

** Nombre de germes < 1 par ml de la phase aqueuse. Number of micro-organisms $<1$ per ml of aqueous phase. 
modérée (FHM), reste à des niveaux de population relativement bas, et elle peut même être inférieure à 1 UFC par $\mathrm{ml}$ dans certains échantillons $\left(\mathrm{B}_{17}, \mathrm{~B}_{18}\right.$, $\mathrm{B}_{19}$ et $\left.\mathrm{B}_{20}\right)$. La FMAT varie de $\mathrm{N}<1$ par $\mathrm{ml}$ à $1 \cdot 10^{5}$ par $\mathrm{ml}$ de la phase aqueuse, la FP de $\mathrm{N}<1$ par ml à $7 \cdot 10^{2}$ par ml et la $\mathrm{FHM}$ de $\mathrm{N}<1$ par $\mathrm{ml}$ à $1,9 \cdot 10^{4}$ par $\mathrm{ml}$.

La flore de contamination d'origine fécale, qui comprend les entérobactéries totales, les bactéries coliformes et les entérocoques, ainsi que la flore pathogène, représentée par les anaérobies sulfito-réducteurs, les staphylocoques présumés pathogènes et les salmonelles, étaient absentes dans tous les échantillons.

Les moisissures et les levures sont présentes en nombres qui peuvent être considérés comme plus importants que les autres flores, mais ceux-ci varient considérablement d'un échantillon à l'autre, allant de $\mathrm{N}<1$ à $7,3 \cdot 10^{6}$ par $\mathrm{ml}$ pour les moisissures et de $\mathrm{N}<1$ à $3,7 \cdot 10^{7}$ par ml pour les levures.

\section{Aspects qualitatifs}

Cent trente souches dont 80 isolées à partir de la FMAT et 50 de la FHM ont fait l'objet d'une identification. Des tests préliminaires nous ont permis de reconnaître parmi ces souches, 125 Bacillus et 5 Staphylococcus.

Parmi les 78 souches de Bacillus, isolées à partir de la FMAT, s'identifient 19 souches de $B$. alvei $(24,3 \%), 17$ souches de $B$. firmus $(12,9 \%), 13$ souches de $B$. brevis $(16,8 \%), 10$ souches de $B$. cereus $(12,9 \%), 10$ souches de $B$. megaterium $(12,9 \%)$, le reste étant représenté par $B$. coagulans (3 souches) et $B$. sphaericus ( 1 souche). 5 autres souches n'ont pu être identifiées.

Les 47 souches isolées de la FHM se répartissent en 31 souches de $B$. alvei $(65,9 \%)$, 11 souches de $B$. brevis $(23,4 \%), 3$ souches de $B$. cereus $(6,4 \%)$ et 2 souches de $B$. firmus $(4,2 \%)$.

Sur l'ensemble des 125 souches qui regroupent les deux flores, les deux espèces $B$, alvei et $B$. brevis prédominent nettement.

\section{B. Etude du smen au cours de son élaboration}

Cette étude a été réalisée sur deux lots de smen A et B, les mêmes que ceux que nous avons étudié du point de vue chimique (El. Marrakchi et al., 1986).

Des prélèvements sont effectués à $\mathbf{J}_{0}$ (beurre avant salage), $\mathrm{J}_{7}$ ( $7^{\mathrm{c}}$ jour après salage), $\mathbf{J}_{40}, \mathbf{J}_{71}$ et $J_{117}$, pour les analyses microbiologiques.

\section{Aspects quantitatifs}

Les résultats obtenus dans le dénombrement de la flore naturelle (FMAT et FP), de la flore de contamination d'origine fécale (coliformes, entérocoques et anaérobies sulfito-réducteurs) et de la flore pathogène (salmonelles et staphylocoques présumés pathogènes) sont résumés dans le tableau 2.

A $\mathbf{J}_{0}$, la FMAT est de $3,8 \cdot 10^{7}$ par $\mathrm{ml}$ pour l'échantillon $\mathrm{A}$ et de $3,1 \cdot 10^{7}$ 
TABleau 2

Evolution de la flore du smen* (nombre de micro-organismes par $\mathrm{ml}$ de la phase aqueuse) Evolution of microbial flora of smen * (CFU/ml of aqueous phase)

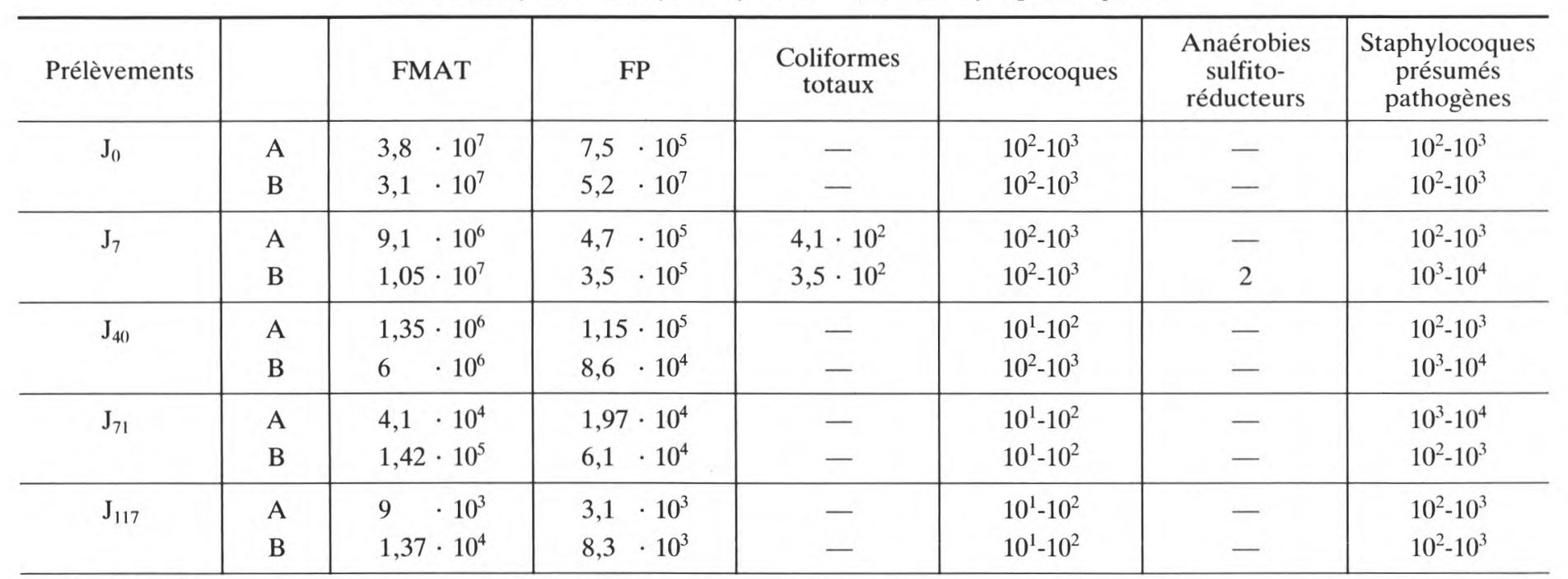

* Absence de coliformes fécaux et de salmonelles dans tous les échantillons.

* Absence of fecal coliforms and salmonella in all the samples.

- Absence. 
par $\mathrm{ml}$ pour $\mathrm{B}$. Elle diminue pendant la conservation pour descendre à $\mathbf{J}_{117}$, à $1,37 \cdot 10^{4}$ par $\mathrm{ml}$ pour B et $9 \cdot 10^{3}$ pour A.

La FP est moins importante que la précédente mais elle évolue de la même façon. A $\mathbf{J}_{0}$, elle est de $7,5 \cdot 10^{5}$ par $\mathrm{ml}$ pour $\mathrm{A}$ et $5,2 \cdot 10^{5}$ par $\mathrm{ml}$ pour B. A $\mathrm{J}_{117}$, elle n'est plus que de $3,1 \cdot 10^{3}$ par ml pour A et $8,3 \cdot 10^{3}$ par ml pour B.

Les coliformes totaux ne sont présents qu'à $J_{7}$ et en nombres assez faibles : 410 coliformes par $\mathrm{ml}$ pour $\mathrm{A}$ et 350 coliformes par $\mathrm{ml}$ pour $\mathrm{B}$, alors que les coliformes fécaux sont absents dans tous les prélèvements. Le nombre des entérocoques est pour les deux échantillons de $10^{2}$ à $10^{3}$ par $\mathrm{ml}$ à $\mathbf{J}_{0}$ et de $10^{1}$ à $10^{2}$ par ml à $\mathrm{J}_{117}$.
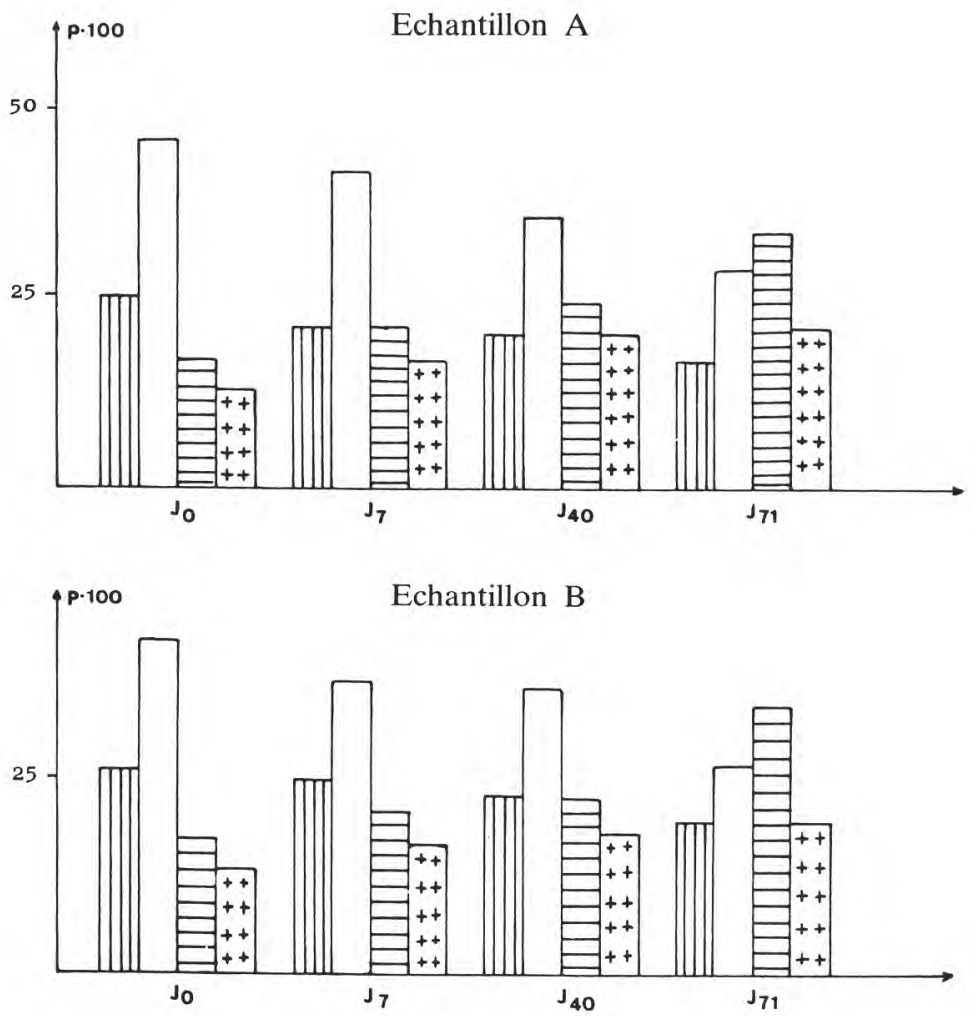

HIS. 1

Identification et évolution des entérocoques.

Identification and evolution of enterococci.

Streptococcus faecalis var. zymogenes

S. faecalis var. liquefaciens

S. faecium 
Les anaérobies sulfito-réducteurs sont absents dans tous les échantillons sauf pour l'échantillon $\mathrm{B}$ où, à $\mathrm{J}_{7}$, leur nombre est de 2 par $\mathrm{ml}$.

Les staphylocoques présumés pathogènes sont présents à des niveaux proches de $10^{2}$ à $10^{3}$ par ml pour les deux échantillons à $\mathrm{J}_{0}$. Leur nombre passe à $10^{3}-10^{4}$ par ml à $\mathrm{J}_{7}$ et à $\mathrm{J}_{40}$ pour l'échantillon $\mathrm{B}$ et il se stabilise à $10^{2}-10^{3}$ par $\mathrm{ml}$ à $\mathrm{J}_{71}$ et $\mathrm{J}_{117}$, dans les deux échantillons.

Les salmonelles sont absentes dans tous les prélèvements, compte tenu du volume ensemencé.

\section{Aspects qualitatifs}

L'identification a porté sur des souches isolées parmi les coliformes (60 souches) et les entérocoques (192 souches).

Dans le groupe des coliformes totaux (tabl. 3), les 5 genres sont représentés. L'espèce Escherichia coli prédomine ( $30 \%$ de l'ensemble des souches), viennent ensuite, les espèces Enterobacter aerogenes (21,6\%), Klebsiella ozaenae $(16,6 \%)$, Hafnia alvei $(8,3 \%)$, E. cloacae $(8,3 \%)$, Citrobacter freundii $(6,6 \%), C$. diversus $(4,9 \%)$ et E. agglomerans $(3,3 \%)$.

La répartition en espèces est presque similaire dans les deux échantillons.

Dans le groupe des entérocoques (histogramme 1), l'espèce Streptococcus faecalis var. liquefaciens prédomine dans les deux échantillons à $\mathbf{J}_{0} ;$ viennent ensuite, les espèces $S$. faecalis var. zymogenes, $S$. faecium et $S$. durans. De $\mathrm{J}_{0}$ à $\mathrm{J}_{71}$, le nombre de $S$. faecium augmente et l'espèce devient alors dominante. Les proportions des espèces $S$. faecalis diminuent mais elles demeurent néanmoins importantes. Quant au nombre de $S$. durans, il augmente sensiblement.

La répartition en espèces et leur évolution dans le temps est la même pourles deux échantillons.

TABLEAU 3

Identification des coliformes

Coliforms identification

\begin{tabular}{l|c|c|c}
\hline \multirow{2}{*}{\multicolumn{1}{c|}{ Espèces identifiées }} & \multicolumn{2}{|c}{ Echantillon } & \multirow{2}{*}{$\begin{array}{c}\text { Nombre } \\
\text { de } \\
\text { souches }\end{array}$} \\
\cline { 2 - 3 } & A & B & 18 \\
\hline Escherichia coli & 10 & 8 & 5 \\
Hafnia alvei & 4 & 1 & 13 \\
Enterobacter aerogenes & 5 & 8 & 5 \\
E. cloacae & 1 & 4 & 2 \\
E. agglomerans & 1 & 1 & 10 \\
Klebsiella ozaenae & 6 & 4 & 4 \\
Citrobacter freundii & 2 & 2 & 3 \\
C. diversus & 1 & 2 & 60 \\
\hline Nombre de souches & 30 & 30 & \\
\hline
\end{tabular}




\section{Discussion}

\section{A. Aspects quantitatifs}

Il y a dans les échantillons de smen du commerce, et pour une même flore, des variations considérables de l'un à l'autre (tabl. 1). Cette hétérogénéité peut être due à leur âge différent. Les divergences sont particulièrement nettes pour les levures (de $\mathrm{N}<1$ à $3,7 \cdot 10^{7}$ par $\mathrm{ml}$ de la phase aqueuse) et les moisissures (de $\mathrm{N}<1$ à $7,3 \cdot 10^{6}$ par $\mathrm{ml}$ ). Hormis les groupes précités, la flore microbienne du smen est, par ailleurs, peu importante.

Toutes les flores étudiées diminuent considérablement au cours de la conservation du produit (tabl. 2).

Cette diminution ne peut être attribuée à l'effet du salage seul. En effet, les teneurs en sel les plus élevées sont de 4 à $5 \%$ de la phase aqueuse à $\mathrm{J}_{7}$ et elles ne peuvent exercer une inhibition aussi importante. Bien plus, ces teneurs baissent au cours de la maturation (El Marrakchi et al., 1986). Shehata et al. (1978), en étudiant l'effet de la consentration en sel sur la conservation du beurre, trouvent, malgré un entreposage à $0^{\circ} \mathrm{C}$, après trois mois, un nombre de micro-organismes appartenant à la FMAT et à la FP augmenté, généralement, d'une puissance de 10 , et ceci, indépendamment de la concentration en chlorure qui peut atteindre dans l'échantillon le plus salé $17 \%$ environ, dans la phase aqueuse. Dans le cas du smen, compte tenu du mode de préparation (maturation spontanée du lait, absence de traitement thermique et entreposage à température ambiante), l'inhibition des micro-organismes résulte de l'action conjuguée des acides gras libres (AGL) et du chlorure de sodium. La baisse des teneurs en lactose et en protéines, observée pendant l'élaboration du produit (El MARRAKCHI et al., 1986), peut aussi, d'une façon secondaire, défavoriser le développement microbien mais seulement en fin de maturation. L'effet inhibiteur des AGL apparaît nettement dans les échantillons $B_{17}, B_{18}$, $\mathrm{B}_{19}$ et $\mathrm{B}_{20}$ où toutes les flores sont absentes et les indices d'acide sont les plus élevés respectivement 52,13 - 68,45 - 84,15 et 82,47 (tabl. 1).

L'absence totale d'entérobactéries dans le smen du commerce ainsi que la disparition rapide des coliformes totaux dans le produit au cours de son élaboration peuvent s'expliquer par l'effet inhibiteur des AGL. SPECTOR (1946) a constaté, le premier, l'effet d'opposition d'un mélange d'acides gras du beurre sur une culture d'Escherichia coli. Cet effet est limité, dans la majorité des cas, aux acides gras à courte et moyenne chaîne, de $\mathrm{C}_{4}$ à $\mathrm{C}_{12}$ (FreESE et al., 1973 ; FAY et FARIAS, 1975). L'action résulterait de l'accumulation de ces composés diffusibles à l'intérieur des cellules bactériennes qui ne peuvent les métaboliser en milieu anaérobie, alors qu'en aérobiose les cellules ne sont pas affectées (RITTENBerg et al., 1950).

L'absence des germes pathogènes recherchés (Staphylocoques présumés entérotoxinogènes et salmonelles) montre que le smen, malgré les nombreuses manipulations que nécessite sa préparation, présente une garantie sanitaire pour le consommateur. 


\section{B. Aspects qualitatifs}

\section{Microflore du smen}

La flore globale (FMAT et FHM) est essentiellement représentée par les bactéries gram-positif. Au sein de ce groupe, le genre Bacillus prédomine ; il représente $96 \%$ de la flore. Le reste est constitué par des staphylocoques.

La prédominance des Bacillus dans la microflore du smen s'explique par leur résistance à certains facteurs d'inhibition. Certaines espèces peuvent tolérer des concentrations en chlorure de sodium de $25 \%$ et résister à des valeurs de $\mathrm{pH}$ égales à 2 (Buchanan et Gibbons, 1974). Ils possèdent aussi une résistance à l'effet défavorable des acides gras libres (NIEMAN, 1954).

\section{Flore d'intérêt hygiénique}

Les coliformes totaux, isolés uniquement à $\mathrm{J}_{7}$, sont essentiellement représentés par Escherichia coli, espèce qui prédomine dans le groupe des coliformes fécaux. Or, ces derniers n'ont été, à aucun moment, isolés. La température de $44{ }^{\circ} \mathrm{C}$, habituellement utilisée pour leur recherche et leur dénombrement peut être dysgénésique pour nos souches. En effet, pour Menlman et al. (1974), aux températures de $45,5^{\circ} \mathrm{C}$ et $44^{\circ} \mathrm{C}$, 75 et $50 \%$ respectivement des souches d'E. coli, sont inhibées. Toutes les variétés thermosensibles appartiennent, cependant, à des sérotypes pathogènes, soit pour l'homme soit pour l'animal. En ce qui concerne les souches isolées à $\mathbf{J}_{7}$, des essais de culture en bouillon lactosé aux températures de 30, 37, 40 et $44^{\circ} \mathrm{C}$ pendant $48 \mathrm{~h}$ ont prouvé qu'aucun développement n'a été possible à $44^{\circ} \mathrm{C}$ pendant 24 à $48 \mathrm{~h}$, alors qu'aux autres températures étudiées toutes les souches se sont multipliées.

Parmi les entérocoques, Streptococcus faecalis var. liquefaciens prédomine largement au niveau du beurre et pendant les premières semaines de la maturation du smen. Cette évolution peut s'expliquer par le fait que la croissance de $S$. faecalis est peut-être stimulée par les acides gras à courte chaîne (butyrique, caproïque et caprique) et empêchées par les acides gras à nombre de carbone plus élevé (CHANDER et al., 1979). Or, nous avons montré que les acides gras à courte chaîne sont libérés pendant les premières semaines de la conservation; viennent ensuite les acides gras insaturés puis les acides gras saturés (EL MARRAKCH et al., 1986). S. faecalis var. liquefaciens représente l'espèce dominante des entérocoques du fromage de Roquefort où le salage et la lipolyse sont également importants (Devoyod, 1969). 


\section{Références bibliographiques}

Buchanan R.E., Gibbons N.E., 1974. Bergey's Manual of Determinative Bacteriology. $8^{\mathrm{e}}$ ed. The Williams and Wilkins Company, Baltimore.

Buttiaux R., Beeren H., Tacquet A., 1974. Manuel de techniques bactériologiques. Ed. Méd. Flammarion, Paris.

Chander H., Ranganathan B., Singh J., 1979. Role of some fatty acids on the growth and the production of lipase by Streptococcus faecalis. J. Food Sci., 44, 1566-1567.

Deeth H.C., Fitz-Gerald C.H., 1976. Lipolysis in dairy products; a review. Aust. J. Dairy Technol., 31, 53-64.

Devoyod J.J., 1969. La flore microbienne du fromage de Roquefort. IV. Les entérocoques. Lait, 49, 637-649.

El Marrakchi A., Berrada M., Chahboun M., Benbouhou M., 1986. Etude chimique du smen marocain. Lait, 66 (2), 117-133.

FAY J.P., Farias R.N., 1975. The inhibitory action of fatty acids on the growth of Escherichia coli. J. Gen. Microbiol., 91, 233-240.

Freese E., Shew C.W., Galliers E., 1973. Fonction of lipophilic acids as antimicrobial food additives. Nature London, 241, 321-325.

Gordon R.E., Haynes W.C., Hor-NAy-PANG C.C., 1973. The genus Bacillus. Agriculture Handbook, $\mathrm{n}^{\circ}$ 427. Agriculture Research Service. United States, Department of Agriculture.

Knight B.C.J.G., Рroom H., 1950. A comparative survey of the nutrition and physiology of mesophilic species in the genus Bacillus. J. Gen. Microbiol., 4, 508.

LE MinOR L., 1972. Le diagnostic de laboratoire des bacilles à gram-négatif. Entérobactéries. Ed. de la Tourelle, Saint-Mandé.

Mehlman I.J., Simon N.T., Snaders A.C., Olson J.C., 1974. Problems of the recovery and identification of enteropathogenic Escherichia coli from foods. J. Milk Food Technol., 37, 350356.

NiEman C.R., 1954. Influence of trace amounts of fatty acids on the growth of micro-organisms. Bacteriol. Rev., 18, 147-163.

Norris J.R., Berkeley R.C.W., Logan N.A., O'Donnel A.G., 1980. The genus Bacillus. In : The prokaryotes. A handbook on habitats, Isolation and Identification. Ed. Mortimer, New York, 1713-1714.

Serres L., Amariglio S., Petransxiene D., 1973. Contrôle de la qualité des produits laitiers. Tome II. Analyse microbiologique. Direction des Services Vétérinaires, France.

Shehata A.E., Magdoub M.I., El Samgary Y.A.A., Hassan A.A., 1978. Effet of salt concentration on the butter flora. Milchwissenschaft, 33, 292-294.

SPECTOR H., 1946. The comparative effect of the butter fat and corn oil on the growth and metabolism of micro-organism. Arch. Biochem., 11, 167-177.

Wolf J.P., 1968. Manuel d'analyse des corps gras. Ed. Azoulay, Paris. 